\title{
Long-term survival after hepatectomy for metachronous liver metastasis of pancreatic ductal adenocarcinoma: a case report
}

\author{
Chikanori Tsutsumi ${ }^{1}$, Toshiya Abe ${ }^{1 *} \mathbb{D}$, Tomohiko Shinkawa ${ }^{1}$, Kazuyoshi Nishihara $^{1}$, Sadafumi Tamiya ${ }^{2}$ and
} Toru Nakano ${ }^{1}$

\begin{abstract}
Background: Pancreatic ductal adenocarcinoma (PDAC) is among the most aggressive malignancies. The prognosis for recurrence after surgery is extremely unfavorable, and liver metastasis of PDAC confers poor prognosis despite resection.

Case presentation: A 51-year-old man was admitted to our hospital for further examination and treatment, including surgery for a pancreatic tumor. On close inspection, he was suspected to have pancreatic head cancer without enlarged lymph nodes or distant metastasis, and pancreatoduodenectomy with D2 lymph node dissection was performed. A postoperative pathological examination revealed well-differentiated invasive ductal adenocarcinoma with lymph node metastasis (stage IIB; pT2N1M0). Postoperatively, he received adjuvant chemotherapy containing gemcitabine for 1 year. Eight years after the radical surgery, his serum carbohydrate antigen 19-9 level was elevated, and computed tomography (CT) and magnetic resonance imaging revealed a well-circumscribed 10-mm mass in liver segment 5. Positron emission tomography/CT also revealed high fluorine-18-fluorodeoxyglucose uptake only in this hepatic tumor. Accordingly, the patient was diagnosed with a solitary liver metastasis of PDAC. As the liver metastasis was isolated and identified long after the initial surgery, we decided to resect it using laparoscopic partial hepatectomy of segment 5. Histopathological examination confirmed liver metastasis of PDAC and the patient received adjuvant chemotherapy containing S-1. No evidence of recurrence has been seen for 11 years since the pancreatoduodenectomy and 3 years since the hepatic resection.

Conclusions: Cases of metachronous liver metastasis of PDAC after radical surgery, in which patients exhibit long-term survival without recurrence after hepatectomy, are extremely rare. Hepatectomy may confer long-term survival, and the time to postoperative recurrence and the number of liver metastases may be useful criteria for deciding whether to perform hepatic resection.
\end{abstract}

Keywords: Hepatectomy, Long-term survival, Liver metastasis, Pancreatic ductal adenocarcinoma

\footnotetext{
* Correspondence: t-abe@surg1.med.kyushu-u.ac.jp

${ }^{1}$ Department of Surgery, Kitakyushu Municipal Medical Center, 2-1-1 Bashaku,

Kokurakita-Ku, Kitakyushu 802-0077, Japan

Full list of author information is available at the end of the article
}

\section{Springer Open}

(c) The Author(s). 2020, corrected publication [2020]. Open Access This article is licensed under a Creative Commons Attribution 4.0 International License, which permits use, sharing, adaptation, distribution and reproduction in any medium or format, as long as you give appropriate credit to the original author(s) and the source, provide a link to the Creative Commons licence, and indicate if changes were made. The images or other third party material in this article are included in the article's Creative Commons licence, unless indicated otherwise in a credit line to the material. If material is not included in the article's Creative Commons licence and your intended use is not permitted by statutory regulation or exceeds the permitted use, you will need to obtain permission directly from the copyright holder. To view a copy of this licence, visit http://creativecommons.org/ licenses/by/4.0/. 


\section{Background}

Pancreatic ductal adenocarcinoma (PDAC) is among the most aggressive malignancies. Despite recent progress in diagnostic imaging modalities, surgical procedures, and chemotherapy, its prognosis remains poor [1, 2]. Here, we report an extremely rare case of metachronous liver metastasis of PDAC after pancreatoduodenectomy (PD) in a patient who survived without a recurrence for 11 years after PD and 3 years after hepatectomy.

\section{Case presentation}

A 51-year-old man was admitted to our hospital for further examination and treatment, including surgery for a pancreatic tumor, detected using abdominal ultrasound (US) and computed tomography (CT). He had no history of malignancy, and physical and laboratory examinations, including tumor markers, revealed no specific findings. Contrast-enhanced CT and gadolinium-ethoxybenzyldiethylenetriamine pantaacetic acid (Gd-EOB-DPTA)-enhanced magnetic resonance imaging (MRI) revealed a nodule that was gradually contrasted in the pancreatic head (Fig. 1a-d). No enlarged lymph nodes or distant metastases were evident. Endoscopic retrograde cholangiopancreatography showed no pancreatic duct dilation or irregularity. Pancreatic juice cytology showed no malignancy. Endoscopic US revealed a well-defined hypoechoic $19 \times 13 \mathrm{~mm}$ mass on the ventral side of the pancreatic head. Accordingly, pancreatic cancer was suspected and PD with D2 lymph node dissection was performed. A macroscopic examination of the resected specimen showed a well-circumscribed $21 \times 15 \mathrm{~mm}$ nodule in the pancreatic head (Fig. 2). A postoperative pathological examination revealed a well-differentiated invasive ductal adenocarcinoma with lymphatic invasion and lymph node metastasis (N1; 1/19) (Fig. 3). The tumor was classified as stage IIB (pT2N1M0) according to the 8th edition of the International Union Against Cancer Tumor Node Metastasis classification. The patient experienced postoperative pancreatic fistula (Clavien-Dindo grade IIIa) [3], which was treated with drainage and antibiotic agents, and was discharged on postoperative day 50 . Two months after the surgery, adjuvant chemotherapy containing gemcitabine was administered at a dose of $1700 \mathrm{mg}\left(1000 \mathrm{mg} / \mathrm{m}^{2}\right)$ on days 1,8 , and 15 every 4 weeks for 1 year. Thereafter, the carbohydrate antigen 19-9 (CA19-9) level was within the normal range and a follow-up CT revealed no local recurrence or distant metastasis. However, at 8 years after the first surgery, the serum CA19-9 level was elevated (130.3 $\mathrm{U} / \mathrm{mL}$ ). Additionally, CT identified a $10-\mathrm{mm}$ low-density area in liver segment 5 (Fig. 4a), while Gd-EOB-DPTAenhanced MRI revealed a well-defined mass in the area (Fig. 4b). Positron emission tomography/CT also revealed high fluorine-18-fluorodeoxyglucose uptake only in this hepatic tumor (Fig. 4c). Furthermore, upper and lower gastrointestinal endoscopy revealed no malignant findings. No other distant metastases were observed. Accordingly, he was diagnosed with liver metastasis of PDAC. Because the liver metastasis was isolated long after the initial

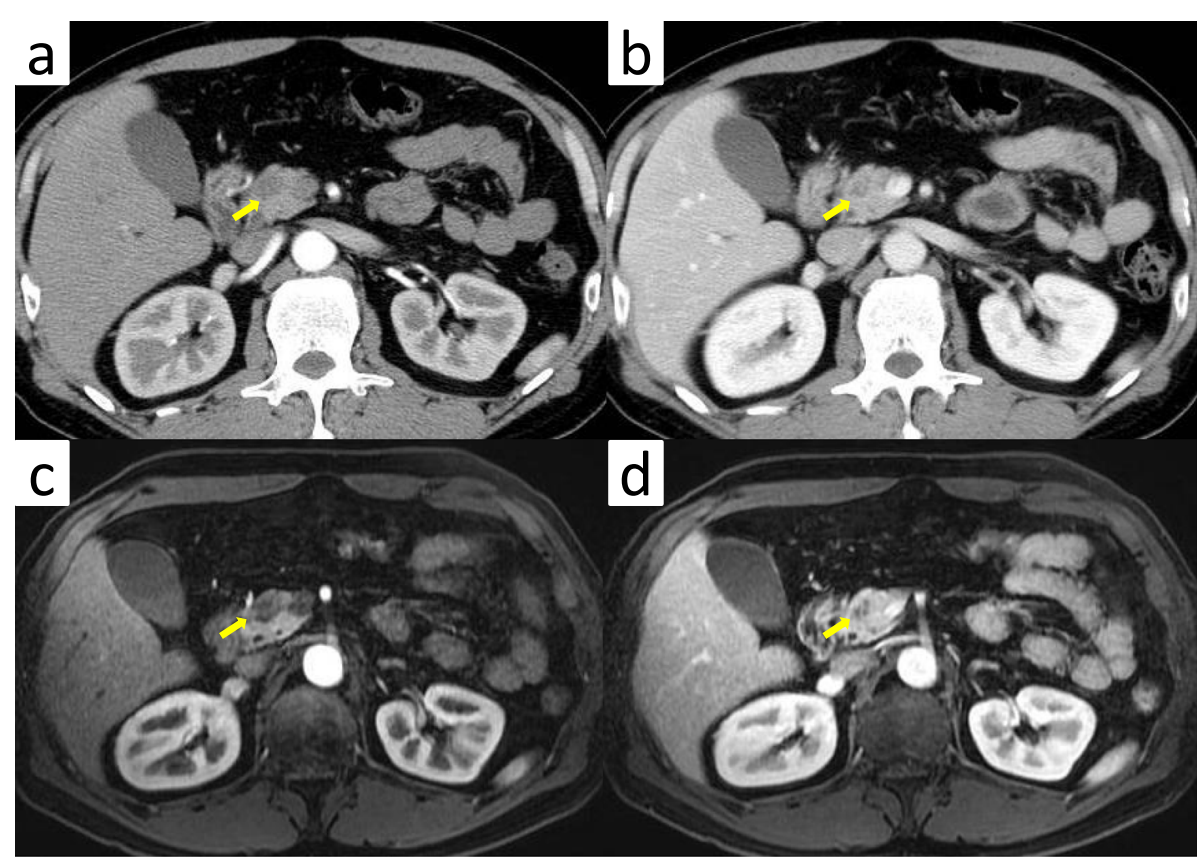

Fig. 1 Contrast-enhanced CT and Gd-EOB-DPTA-enhanced MRI revealed a nodule that was gradually contrasted in the pancreatic head (arrow). a Arterial phase of $C$. $\mathbf{b}$ Portal phase of $C$. $\mathbf{c}$ Early phase of MRI. $\mathbf{d}$ Late phase of MRI. $C$, computed tomography; Gd-EOB-DPTA, gadolinium-ethoxybenzyl-diethylenetriamine; $M R l$, magnetic resonance imaging 


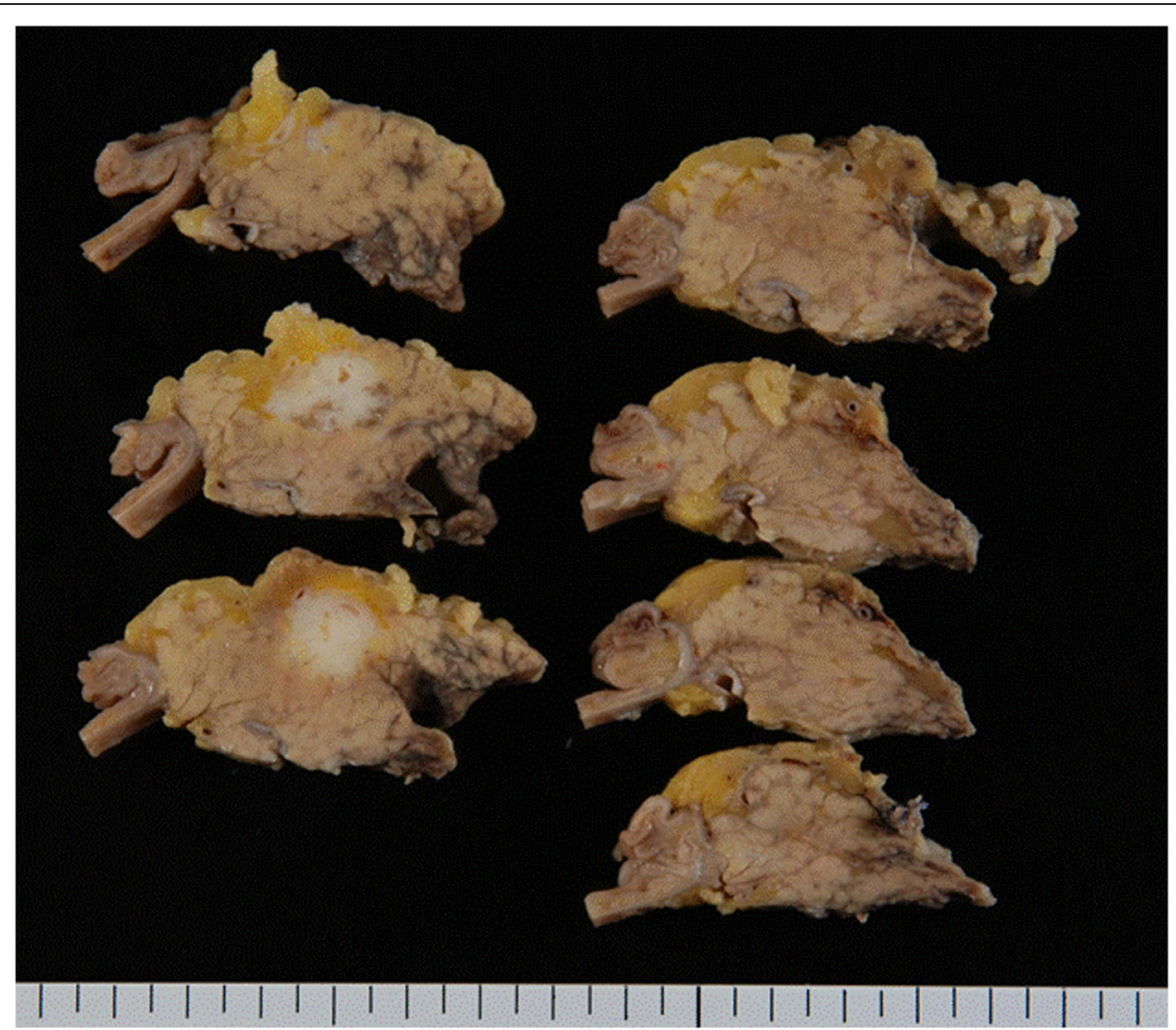

Fig. 2 Macroscopic examination of the resected specimen identified a well-circumscribed $21 \times 15 \mathrm{~mm}$ nodule in the pancreatic head (arrow)

surgery, we decided to resect it using laparoscopic partial hepatectomy of segment 5 at 8 years and 1 month after the PD. A macroscopic examination of the resected specimen revealed a $10 \times 9 \mathrm{~mm}$ nodular tumor under the liver subcapsular region (Fig. 5). A postoperative pathological examination demonstrated well to moderately differentiated adenocarcinoma (Fig. 6a, b) with no continuity between the liver tumor and the peripheral bile duct. Additionally, immunostaining was positive for cytokeratin 17 (CK17) and MUC5AC, and the immunostaining

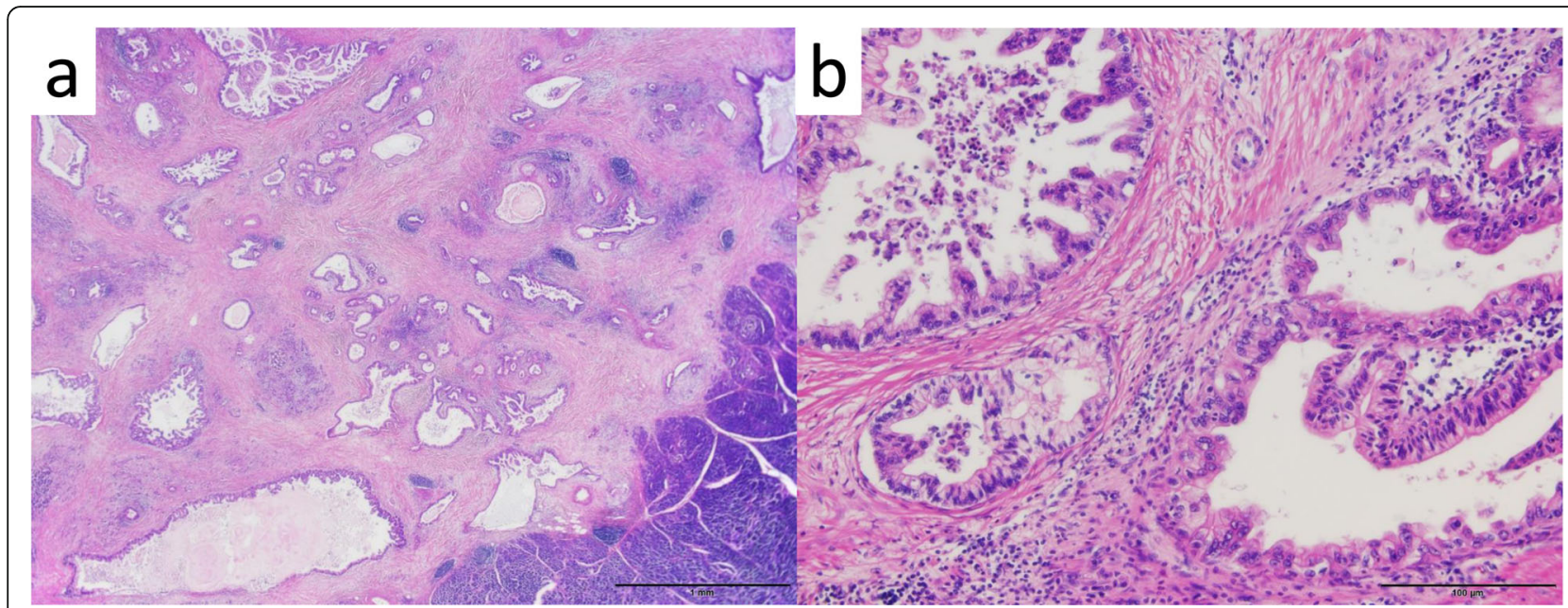

Fig. 3 Histopathological findings (hematoxylin-eosin staining) showing well-differentiated invasive ductal adenocarcinoma with lymphatic invasion. $\mathbf{a} \times 4$ and $\mathbf{b} \times 40$ original magnification 


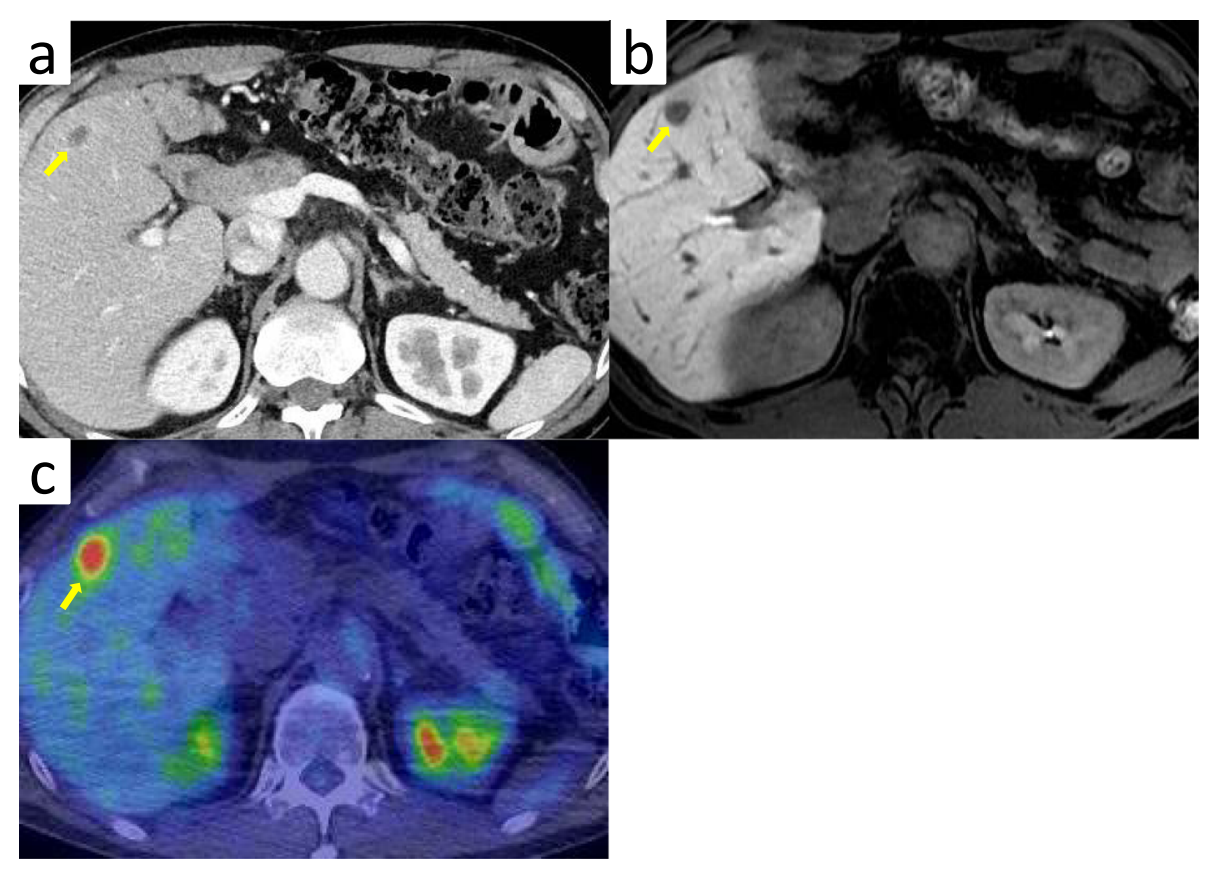

Fig. 4 a Contrast-enhanced CT showing a 10-mm low-density area in liver segment 5 (arrow). b Gd-EOB-DPTA-enhanced MRI showing a welldefined mass in liver segment 5 (arrow). c PET/CT showing the liver mass with high fluorine-18-fluorodeoxyglucose uptake (arrow). CT, computed tomography; Gd-EOB-DPTA, gadolinium-ethoxybenzyl-diethylenetriamine; MRI, magnetic resonance imaging; PET, positron emission tomography

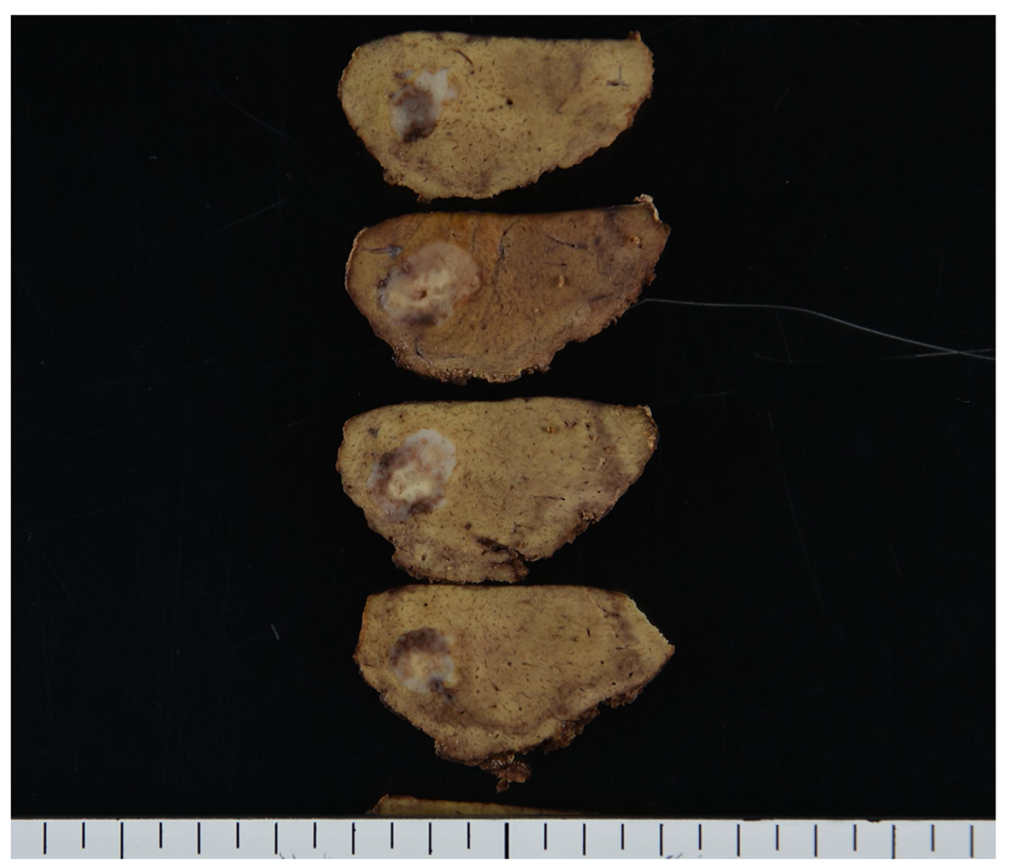

Fig. 5 Macroscopic examination of the revealed specimen showed a nodular $10 \times 9 \mathrm{~mm}$ tumor under the liver subcapsular region 


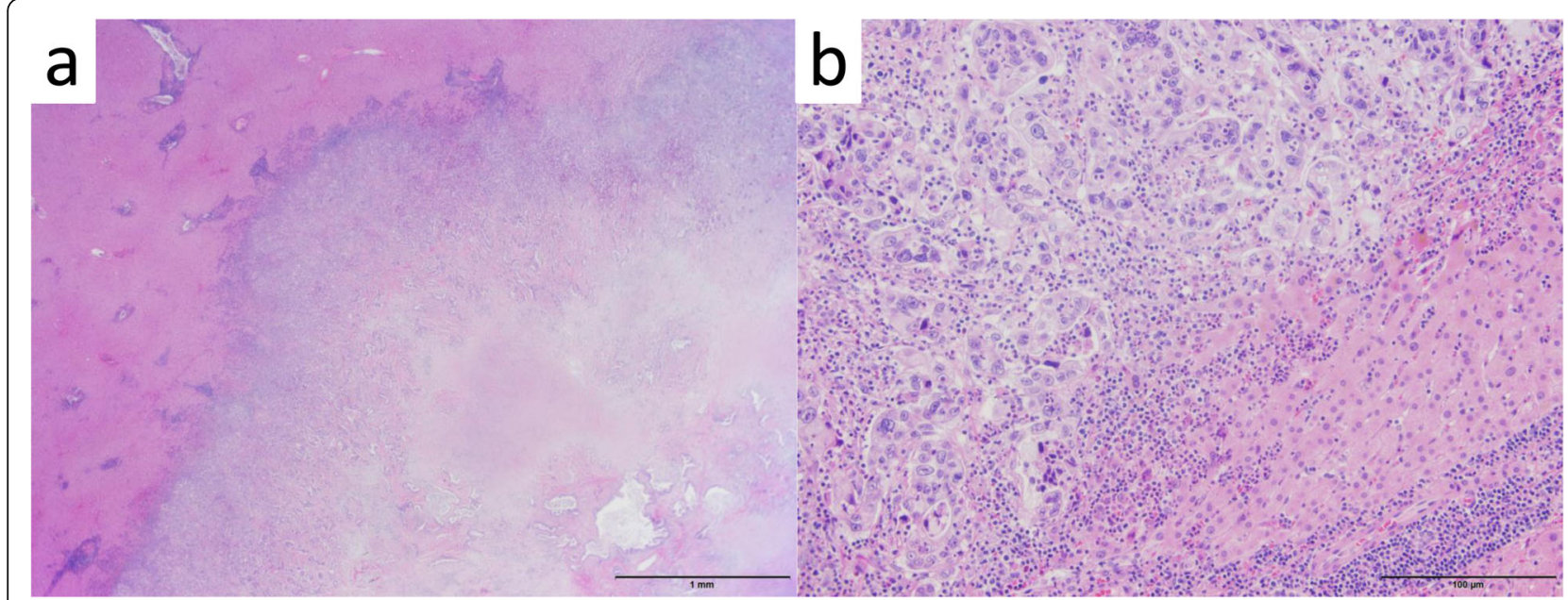

Fig. 6 Histopathological findings (hematoxylin-eosin staining) showing a well to moderately differentiated adenocarcinoma compatible with a metastasis of pancreatic ductal adenocarcinoma. $\mathbf{a} \times 4$ and $\mathbf{b} \times 40$ original magnification

findings of the metastatic lesion were consistent with those of the primary lesion (Fig. 7). Based on the above results, the final pathological diagnosis was liver metastasis of PDAC. We planned to administer chemotherapy for 6 months. However, the patient strongly hoped to continue adjuvant chemotherapy beyond that period. For this reason, he received adjuvant chemotherapy for 1 year. No evidence of recurrence was noted at 11 years after the PD and 3 years after the hepatic resection.

\section{Discussion}

The prognosis of postoperative recurrence of PDAC is extremely poor [4]. A recent guideline for metastatic PDAC recommended intensive chemotherapy regimens such as FOLFIRINOX (fluorouracil, leucovorin, irinotecan, and oxaliplatin) or gemcitabine plus nanoparticle albuminbound paclitaxel as the first-line treatment [5]. For this reason, liver metastases of PDAC are not generally indicated for surgery [6]. Some researchers insist that the resection of

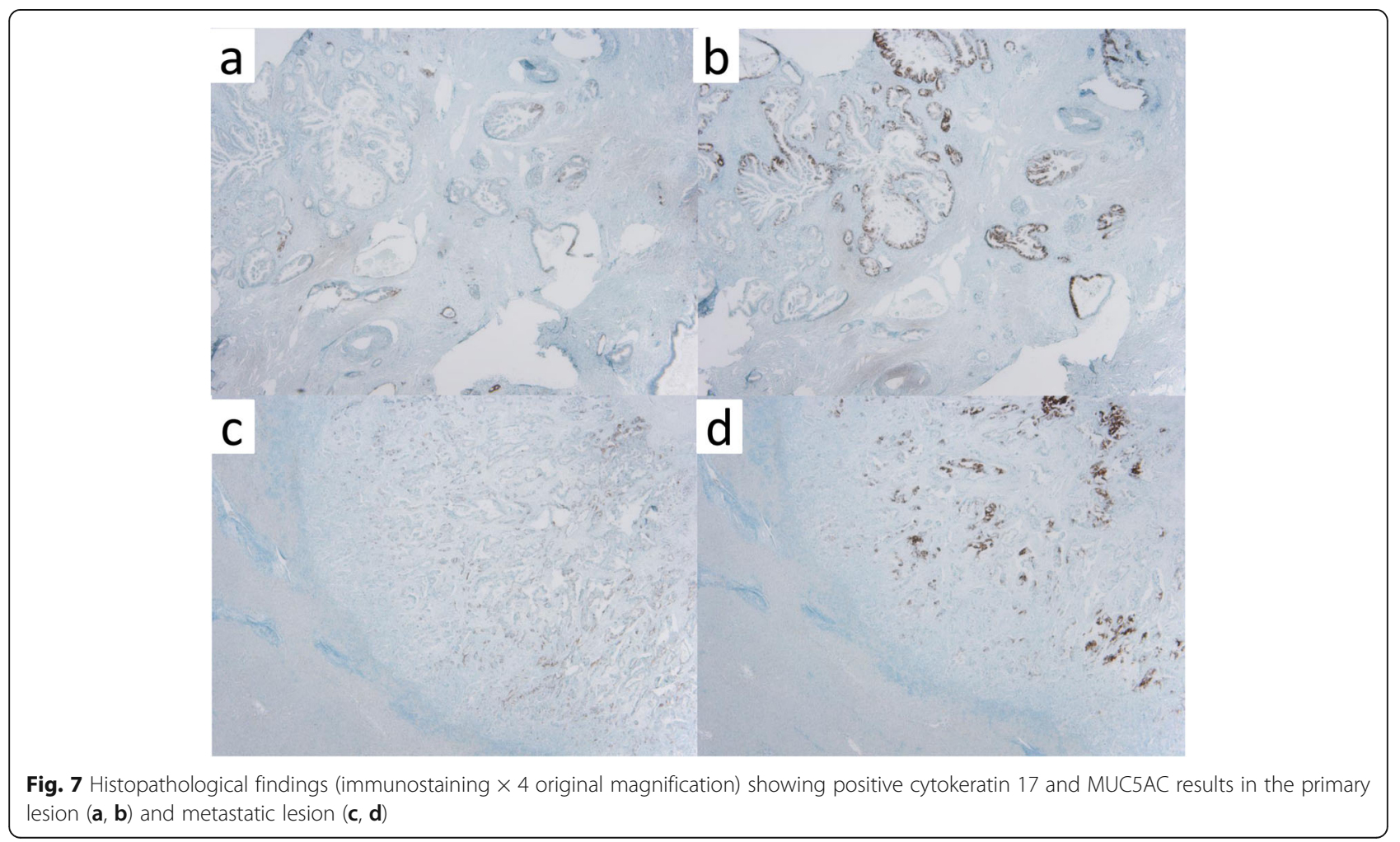




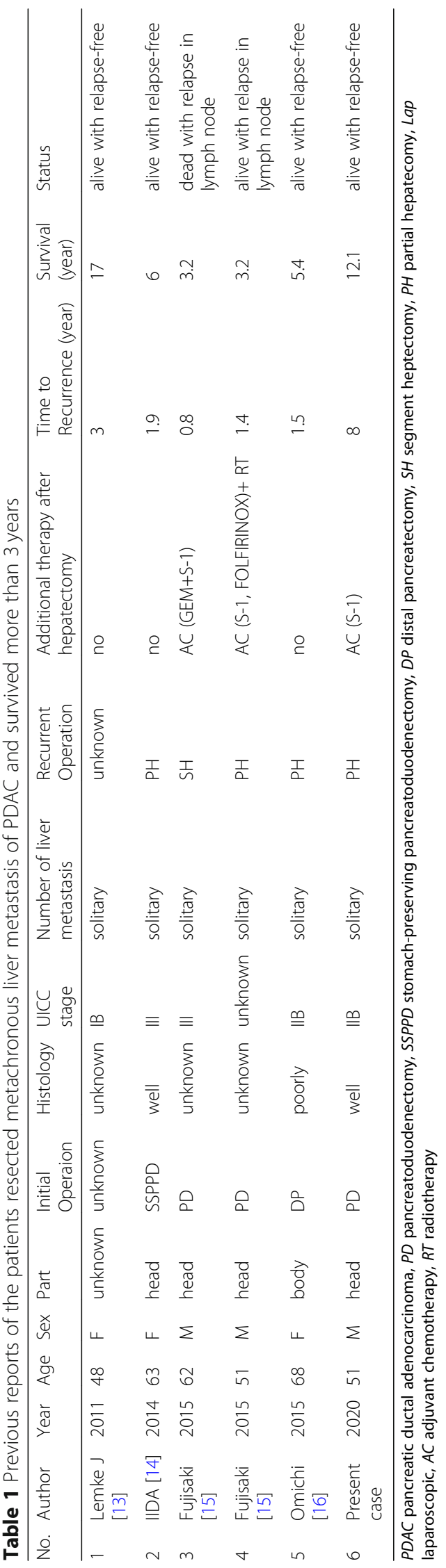


liver metastasis is not beneficial [7], while others suggest the use of hepatectomy in selected patients [8-10]. Because the selection criteria remain vague, patients with liver metastases of PDAC who would benefit from hepatectomy should be identified. At the time of the hepatectomy in the present case, the Japanese clinical practice guidelines for pancreatic cancer 2016 [11] had not yet stated the indication for liver resection for liver metastasis. Thus, in the present case, we selected partial hepatectomy without administering additional chemotherapy after the diagnosis of liver metastasis of PDAC because the liver metastasis was isolated and no recurrence was identified for 8 years after the radical surgery. It is difficult to discriminate intrahepatic cholangiocarcinoma (ICC) from liver metastasis of PDAC due to histological similarities between them. Lok et al. [12] reported higher frequencies of CK17 and MUC5AC in PDAC than in ICC $(60 \%$ vs $12 \%$ and $67 \%$ vs $12 \%$, respectively). In the present case, CK17 and MUC5AC immunostaining findings were positive, and the immunostaining findings of the metastatic lesion were consistent with those of the primary lesion. Additionally, there was no continuity between the liver tumor and the peripheral bile duct. Accordingly, we considered the liver tumor metastatic PDAC. A search of the literature revealed that only 6 patients survived more than 3 years after the initial surgery, hepatectomy for metachronous liver metastasis, including the present case (Table 1) [13-16]. Almost all patients exhibited a relatively long time to relapse, and these results suggest that a longer time to relapse may be associated with a longer survival time. Additionally, all patients had a solitary metastasis. Hackert et al. [9] reported a 5-year survival rate of $8.1 \%$ after surgery for liver oligometastases. Zanini et al. [17] demonstrated that patients with metachronous liver metastasis had a significantly longer median survival than that of patients with synchronous disease (11.4 months vs. 8.3 months, $p=0.038$ ). Furthermore, in a retrospective analysis, overall survival was significantly prolonged in patients with resected liver metastasis of PDAC (median 14 months vs. 8 months, $p<0.001$ ) [10]. These findings indicate that hepatectomy might confer long-term survival when the liver metastasis is isolated and the time to postoperative recurrence is long. In addition, Voss et al. uncovered that the resection of liver metastasis of PDAC can be safely performed [18]. Therefore, for patients with liver metastasis of PDAC, onset timing, number of lesions, and time to recurrence may be useful criteria for surgery. Further studies are needed to determine the indications for liver resection.

PDAC involves the early capability of cells to migrate to distant organs, and many patients have metastatic disease at the time of PDAC diagnosis [19]. For this reason, controlling micrometastases using systemic chemotherapy is important for patient long-term survival. The literature search [13-16] (Table 1) revealed that adjuvant chemotherapy has been used in many cases and that multidisciplinary therapy including chemotherapy may contribute to long-term survival. In PDAC, it is possible for other metastases to appear shortly after the diagnosis, even if none are evident at the time of diagnosis. Therefore, it may be important to determine the effectiveness of hepatectomy after chemotherapy and follow patients for a certain duration.

\section{Conclusions}

Here, we reported an extremely rare case of metachronous liver metastasis of PDAC after radical surgery in a patient who survived without a recurrence for 11 years after PD and 3 years after hepatectomy. The present findings suggested that hepatectomy may confer longterm survival in selected patients. The time to postoperative recurrence and the number of liver metastases may be useful criteria for deciding whether to perform hepatic resection.

\section{Abbreviations \\ CA19-9: Carbohydrate antigen 19-9; CT: Computed tomography; Gd-EOB- DPTA: Gadolinium-ethoxybenzyl-diethylenetriamine pantaacetic acid; ICC: Intrahepatic cholangiocarcinoma; MRI: Magnetic resonance imaging; PD: Pancreatoduodenectomy; PDAC: Pancreatic ductal adenocarcinoma; US: Ultrasound}

\section{Acknowledgements \\ The present study was supported in part by a Japan Society for the Promotion of Science Grant-in-Aid for Research Activity start-up (no. 19 K23918), the Shinni- hon Foundation of Advanced Medical Treatment Research, and the Fukuoka Foundation for Sound Health Cancer Research Fund. \\ We thank Editage (www.editage.com) for English language editing.}

\section{Authors' contributions}

$\mathrm{CT}$ wrote the manuscript. TA supervised the writing of the manuscript. TS and $\mathrm{KN}$ performed the surgical procedures and managed the patient's perioperative course. ST contributed to drafting the pathological findings portion of the manuscript. TN provided final approval of this manuscript. The authors read and approved the final manuscript.

\section{Funding}

Not applicable.

Availability of data and materials

Not applicable.

Ethics approval and consent to participate

Not applicable.

\section{Consent for publication}

Written informed consent was obtained from the patient for the publication of this case report and any accompanying images.

\section{Competing interests}

The authors declare no competing interests.

\section{Author details}

'Department of Surgery, Kitakyushu Municipal Medical Center, 2-1-1 Bashaku, Kokurakita-Ku, Kitakyushu 802-0077, Japan. ²Department of Pathology, Kitakyushu Municipal Medical Center, Kitakyushu, Japan. 
Received: 26 May 2020 Accepted: 25 June 2020

Published online: 03 July 2020

\section{References}

1. Siegel RL, Miller KD, Jemal A. Cancer statistics, 2019. CA Cancer J Clin. 2019; 69:7-34.

2. Michalski CW, Erkan M, Hüser N, Müller MW, Hartel M, Friess $H$, et al. Resection of primary pancreatic cancer and liver metastasis: a systematic review. Dig Surg. 2008;25:473-80.

3. Clavien PA, Barkun J, de Oliveira ML, Vauthey JN, Dindo D, Schulick RD, et al. The Clavien-Dindo classification of surgical complications: five-year experience. Ann Surg. 2009;250:187-96.

4. Jemal A, Siegel R, Xu J, Ward E. Cancer statistics, 2010. CA Cancer J Clin. 2010;60:277-300

5. Conroy T, Desseigne F, Ychou M, Bouché O, Guimbaud R, Bécouarn Y, et al. FOLFIRINOX versus gemcitabine for metastatic pancreatic cancer. N Engl J Med. 2011;364:1817-25.

6. Tempero MA, Arnoletti JP, Behrman S, Ben-Josef E, Benson AB 3rd, Berlin JD, et al. Pancreatic adenocarcinoma, version 2.2012: featured updates to the NCCN guidelines. J Natl Compr Canc Netw. 2012;10:1703-13.

7. Thomas RM, Truty MJ, Nogueras-Gonzalez GM, Fleming JB, Vauthey JN, Pisters PW, et al. Selective reoperation for locally recurrent or metastatic pancreatic ductal adenocarcinoma following primary pancreatic resection. J Gastrointest Surg. 2012;16:1696-704.

8. Shrikhande SV, Kleeff J, Reiser C, Weitz J, Hinz U, Esposito I, et al. Pancreatic resection for M1 pancreatic ductal adenocarcinoma. Ann Surg Oncol. 2007; 14:118-27.

9. Hackert T, Niesen W, Hinz U, Tjaden C, Strobel O, Ulrich A, et al. Radical surgery of oligometastatic pancreatic cancer. Eur J Surg Oncol. 2017:43:358-63.

10. Tachezy M, Gebauer F, Janot M, Uhl W, Zerbi A, Montorsi M, et al. Synchronous resections of hepatic oligometastatic pancreatic cancer: disputing a principle in a time of safe pancreatic operations in a retrospective multicenter analysis. Surgery. 2016;160:136-44.

11. Yamaguchi K, Okusaka T, Shimizu K, Furuse J, Ito Y, Hanada K, et al. Clinical practice guidelines for pancreatic cancer 2016 from the japan pancreas society: a synopsis. Pancreas. 2017;46:595-604.

12. Lok T, Chen L, Lin F, Wang HL. Immunohistochemical distinction between intrahepatic cholangiocarcinoma and pancreatic ductal adenocarcinoma. Hum Pathol. 2014:45:394-400.

13. Lemke J, Barth TF, Juchems M, Kapapa T, Henne-Bruns D, Kornmann M. Long-term survival following resection of brain metastases from pancreatic cancer. Anticancer Res. 2011;31:4599-603.

14. lida T, Nakabayashi Y, Okui N, Shiba H, Otsuka M, Yanaga K. Successful management of metachronous liver metastasis after pancreaticoduodenectomy for pancreatic ductal carcinoma using hepatectomy and chemotherapy: a case report. Anticancer Res. 2014;34: 2417-20.

15. Fujisaki S, Takashi M, Tomita R, Sakurai K, Takayama T. Four cases of hepatectomy for liver metastases of intractable hepatobiliary pancreatic cancers. Gan To Kagaku Ryoho. 2015:42:1451-3.

16. Omichi K, Sakamoto H, Takahashi A, Amikura K, Asayama M, Kurozumi M, et al. A case of successful management of liver metastasis from a pancreatic carcinoma by combined hepatectomy and chemotherapy. J Jpn Surg Assoc. 2015;76:1494-8.

17. Zanini N, Lombardi R, Masetti M, Giordano M, Landolfo G, Jovine E. Surgery for isolated liver metastases from pancreatic cancer. Updates Surg. 2015;67: 19-25.

18. Voss N, Izbicki JR, Nentwich MF. Oligometastases in pancreatic cancer (synchronous resections of hepatic oligometastatic pancreatic cancer: disputing a principle in a time of safe pancreatic operations in a retrospective multicenter analysis). Ann Gastroenterol Surg. 2019:3:373-7.

19. Seton-Rogers S. Navigating uncharted territory. Nat Rev Cancer. 2012;12:151.

\section{Publisher's Note}

Springer Nature remains neutral with regard to jurisdictional claims in published maps and institutional affiliations.

\section{Submit your manuscript to a SpringerOpen ${ }^{\circ}$ journal and benefit from:}

- Convenient online submission

- Rigorous peer review

- Open access: articles freely available online

- High visibility within the field

- Retaining the copyright to your article

Submit your next manuscript at $\boldsymbol{\nabla}$ springeropen.com 